\title{
Adiposity, physical fitness and incident diabetes: the physical activity longitudinal study
}

\author{
P. T. Katzmarzyk • C. L. Craig • L. Gauvin
}

Received: 2 August 2006 / Accepted: 1 November 2006/ Published online: 13 January 2007

(C) Springer-Verlag 2007

\begin{abstract}
Aims/hypothesis The purpose of this study was to investigate the relationships among adiposity, physical activity, physical fitness and the development of diabetes in a diverse sample of Canadians.

Methods The sample included 1,543 adults (709 men and 834 women) from the Canadian Physical Activity Longitudinal Study who were free of diabetes at baseline (1988). Several indicators of adiposity (BMI, waist circumference, WHR, sum of skinfold thicknesses), musculoskeletal fitness (sit-ups, push-ups, grip strength, trunk flexibility), cardiorespiratory fitness (maximal metabolic equivalents [METs]) and leisure-time physical activity levels were measured at baseline. Participants were followed until 2002-2004 for the ascertainment of new cases of diabetes.

Results The 15.5-year cumulative incidence of diabetes was $5.0 \%$ (5.2\% in men, $4.9 \%$ in women). Adiposity and physical fitness, but not physical activity, were significant predictors of diabetes after adjustment for age, sex and several covariates. For each standard deviation of the indicators of adiposity, the risk of diabetes was $99-194 \%$ higher. Conversely, the risk was 70 and $61 \%$ lower for each
\end{abstract}

\section{P. T. Katzmarzyk $(\bowtie)$}

School of Kinesiology and Health Studies and Department of Community Health and Epidemiology, Queen's University,

Kingston, ON K7L 3N6, Canada

e-mail: katzmarz@post.queensu.ca

C. L. Craig

Canadian Fitness and Lifestyle Research Institute,

Ottawa, ON, Canada

L. Gauvin

Department of Social and Preventive Medicine, Groupe de Recherche Interdisciplinaire de Santé and Centre de Recherche Léa-Roback, Université de Montréal,

Montreal, QC, Canada standard deviation of maximal METs and composite musculoskeletal fitness score, respectively. Receiver operating characteristic curve analyses confirmed that neither adiposity nor physical fitness provided a superior prediction of incident diabetes.

Conclusions/interpretation Adiposity and physical fitness were both important predictors of the development of diabetes in this cohort of Canadians.

Keywords Cohort study · Diabetes · Fitness · Lifestyle · Obesity. Physical activity
Abbreviations
CRF cardiorespiratory fitness
MET metabolic equivalent
MSF musculoskeletal fitness
OR odds ratio
PALS physical activity longitudinal study
ROC receiver operating characteristic
$V \mathrm{O}_{2 \max }$ maximal oxygen consumption

\section{Introduction}

Chronic diseases are now the leading cause of death worldwide, and their impact is steadily increasing. In response, WHO has called for the strengthening of national strategies for the prevention of cardiovascular disease, cancer and diabetes [1]. Diabetes is a major contributor to the global burden of chronic disease, and it is estimated that its prevalence may increase by as much as $80 \%$ in some regions over the next 10 years [1]. In Canada, the most recent estimates are that $4.6 \%$ of the population aged 12 years or older have physician-diagnosed diabetes [2], with an associated economic cost of almost $\$ 5$ billion 
annually [3]. The prevention of diabetes has been identified as a national priority and an integral component of Canada's diabetes strategy [4]. Within this context, the identification of predictors of diabetes in the Canadian population is important for the development of prevention strategies.

Physical inactivity and obesity are the two major modifiable risk factors for the development of type 2 diabetes, as well as cardiovascular disease and some cancers [5]. However, the degree to which these risk factors are independently associated with type 2 diabetes is not fully understood. A recent review has concluded that body weight has a greater effect on diabetes than physical inactivity, while physical inactivity may have greater influence on cardiovascular disease than body weight [6]. The degree to which physical inactivity, fitness or adiposity are associated with the development of type 2 diabetes has important implications for the development of preventive interventions and public health messages. Thus, the purpose of this study was to estimate the effects of adiposity, physical activity and physical fitness on incident diabetes in a diverse sample of Canadians.

\section{Subjects and methods}

The Physical Activity Longitudinal Study (PALS) is a cohort study of people who originally participated in the 1981 Canada Fitness Survey and/or the 1988 Campbell's Survey of Well-being in Canada. These surveys were based on a sample of households in Canada in 1981 chosen by Statistics Canada to be representative of the Canadian population. As a household-based design, it excluded approximately $3 \%$ of the population who were living in institutions, hospitals, prisons and Indian reserves. Unfortunately, information on ethnicity was not collected as part of these surveys. Participants were traced and sent a followup self-administered questionnaire between September 2002 and April 2004.

Sample The PALS cohort consists of approximately 4,900 individuals assessed in 2002-2004, and was based on the Canada Fitness Survey sample [7]. Given that information on diabetes was not collected in the 1981 Canada Fitness Survey, the 1988 Campbell's Survey on Well-being in Canada was used as the baseline for the present study. The subsample for this investigation included 1,557 adult participants (18-69 years at baseline) who had data available from 1988 and 2002-2004. Participants who indicated a history of diabetes at baseline (1988) were excluded from further analysis $(n=14)$, yielding a final analysis sample of 1,543 (709 men and 834 women). Sample sizes vary from measure to measure because of missing values for some participants (Table 1). PALS was approved by the Ethics Review Board of the Faculty of Medicine, University of Montreal. All participants provided their written informed consent to participate in both the baseline assessment and the follow-up survey.

Exposure variables The baseline assessment of exposure variables was conducted during household visits in 1988. Direct measurements of adiposity, cardiorespiratory fitness (CRF) and musculoskeletal fitness (MSF) were obtained, and a physical activity and lifestyle questionnaire was administered.

Anthropometric dimensions were taken according to the standardized procedures of the Canada Fitness Survey [8]. Stature was measured with a Harpenden tape to the nearest $0.1 \mathrm{~cm}$ and body mass was measured to the nearest $0.1 \mathrm{~kg}$ using a Seca 770 balance scale (Seca Corporation, Columbia, MD, USA). Waist circumference was measured to the nearest millimetre at the point of noticeable waist narrowing using an anthropometric tape. In cases of indeterminate waist narrowing, waist circumference was measured at the estimated lower level of the 12th or lower floating rib. Hip circumference was measured to the nearest millimetre at the level of the symphysis pubis and point of maximal gluteal protuberance using an anthropometric tape. Skinfold thicknesses at the biceps, triceps, subscapular, suprailiac and medial calf sites were measured on the right side of the body with a Harpenden calliper (British Indicators, Burgess Hill, UK) to the nearest $0.2 \mathrm{~mm}$. The WHR and BMI $\left(\mathrm{kg} / \mathrm{m}^{2}\right)$ were calculated, and the five skinfold thicknesses were summed (SF5) to provide an overall measure of subcutaneous adiposity.

Maximal oxygen consumption $\left(V \mathrm{O}_{2 \max }\right)$ was estimated using a modified version of the Canadian Aerobic Fitness Test (CAFT), and was used as the measure of CRF in this study. The CAFT is a progressive step-test in which participants are asked to perform five bouts of exercise on double $20-\mathrm{cm}$ steps. The cadence was set by a cassette tape with a progressive tempo, and heart rates were recorded after each bout. The beginning level was determined on the basis of the participant's age and sex. If the participant's heart rate was below a threshold value, they were allowed to proceed to the next level for a maximum of five bouts. $V \mathrm{O}_{2 \max }$ (metabolic equivalents [METs]) was estimated from the following equation:

$$
\begin{aligned}
V \mathrm{O}_{2 \max }= & {\left[42.5+\left(16.6\left[V_{2}\right]\right)-(0.12[W])\right.} \\
& -(0.12[H])-(0.24[A])] / 3.5
\end{aligned}
$$

where $V \mathrm{O}_{2}$ is the average oxygen cost of the last completed exercise stage in $1 / \mathrm{min}$, obtained from a standardized table, $W$ is the participant's body weight in $\mathrm{kg}, H$ is the heart rate after the final stage of stepping in beats per min, and $A$ is the participant's age in years. 
Table 1 Characteristics of men and women in the Physical Activity Longitudinal Study at baseline (1988)

\begin{tabular}{|c|c|c|c|c|c|c|}
\hline & \multicolumn{3}{|l|}{ Men } & \multicolumn{3}{|l|}{ Women } \\
\hline & $n$ & Mean & SD & $n$ & Mean & SD \\
\hline Age (years) & 742 & 36.8 & 12.4 & 855 & 37.5 & 12.1 \\
\hline BMI $\left(\mathrm{kg} / \mathrm{m}^{2}\right)$ & 595 & 25.5 & 3.5 & 672 & 24.2 & 4.2 \\
\hline Waist circumference $(\mathrm{cm})$ & 542 & 87.9 & 9.9 & 582 & 75.5 & 10.0 \\
\hline Hip circumference $(\mathrm{cm})$ & 541 & 99.7 & 6.3 & 583 & 99.8 & 8.6 \\
\hline WHR & 541 & 0.88 & 0.06 & 582 & 0.76 & 0.06 \\
\hline Sum of skinfold thicknesses (mm) & 536 & 61.2 & 21.6 & 555 & 80.9 & 28.7 \\
\hline Maximal METs & 423 & 12.5 & 1.6 & 429 & 10.7 & 1.6 \\
\hline Push-ups $(n)$ & 425 & 20.4 & 12.6 & 428 & 15.0 & 10.7 \\
\hline Sit-ups $(n$ per min $)$ & 420 & 28.5 & 12.2 & 428 & 21.2 & 12.2 \\
\hline Grip strength (kg) & 428 & 99.8 & 16.7 & 437 & 56.8 & 10.9 \\
\hline Trunk flexibility $(\mathrm{cm})$ & 418 & 26.6 & 9.3 & 437 & 30.4 & 8.4 \\
\hline Physical activity $\left(\mathrm{kJ} \mathrm{kg}^{-1}\right.$ day $^{-1)}$ & 638 & 13.1 & 12.3 & 759 & 9.2 & 9.5 \\
\hline \multicolumn{7}{|l|}{ Smoking status $(n, \%)$} \\
\hline Non-smoker & $367(49.5)$ & & & $444(51.9)$ & & \\
\hline Current smoker & $181(24.4)$ & & & $207(24.2)$ & & \\
\hline Former smoker & $194(26.2)$ & & & $204(23.9)$ & & \\
\hline \multicolumn{7}{|l|}{ Alcohol consumption $(n, \%)$} \\
\hline Abstainer & $282(38.0)$ & & & $479(56.0)$ & & \\
\hline 1-3 drinks per day & $385(51.9)$ & & & $350(40.9)$ & & \\
\hline 4-5 drinks per day & $41(5.5)$ & & & $21(2.5)$ & & \\
\hline $6+$ drinks per day & $34(4.6)$ & & & $5(0.6)$ & & \\
\hline Parental history of diabetes $(n, \%)$ & $73(9.8)$ & & & $105(12.3)$ & & \\
\hline
\end{tabular}

Indicators of MSF included sit-ups, push-ups, combined left- and right-hand grip strength, and sit-and-reach trunk flexibility. All measurements were made following the standardized procedures of the Canada Fitness Survey [8]. The number of push-ups $(n)$ completed without time limit and the number of sit-ups performed in $60 \mathrm{~s}$ ( $n$ per min) were used as indicators of muscular endurance. For push-ups, male subjects balanced from the toes, while female subjects balanced from the knees. One complete push-up entailed straightening the elbows and returning to touch the nose to the floor, while keeping the back straight. Sit-ups were performed from the supine position, with knees flexed $90^{\circ}$. One complete sit-up entailed curling the trunk from the supine position to touching the elbows to the knees, and returning to the start position. Muscular strength was assessed using grip strength, measured with a Stoelting adjustable dynamometer. Participants held the dynamometer at the level of the thigh in line with the forearm and were instructed to squeeze vigorously so as to exert maximum force. The maximum grip strengths of three trials for each hand were summed to provide a single measure of grip strength $(\mathrm{kg})$. A sit-and-reach test was used to assess trunk flexibility. The participant reached towards the toes, with knees flat on the floor. The test was performed twice, with the maximum value recorded to the nearest $0.5 \mathrm{~cm}$.

Given that there are several dimensions to MSF, it is difficult to capture quantitatively with a single measure- ment. Thus, a composite score was calculated by subjecting the scores for sit-ups, push-ups, grip strength and trunk flexibility to principal components analysis, separately by sex. The first principal components explained $47.9 \%$ of the variance in men and $49.6 \%$ of the variance in women, and were retained for further analysis. The correlations between the original variables and the first principal component can be interpreted as loadings that represent their contributions to the overall score. The factor loadings were $0.50,0.80$, 0.82 and 0.59 in men and $0.41,0.84,0.80$ and 0.68 in women for grip strength, sit-ups, push-ups and trunk flexibility, respectively, indicating that the composite MSF score included positive contributions for each original variable, and was similar in men and women.

Leisure-time physical activity levels were assessed using a questionnaire modelled after the Minnesota Leisure Time Physical Activity Questionnaire [9], which collected information about physical activity levels over the previous 12 months. A list of physical activities was provided and respondents indicated the number of occasions and the average duration of the activity bouts. Average daily leisure-time activity energy expenditure (AEE) was calculated as follows:

$\operatorname{AEE}\left(\mathrm{kJ} \mathrm{kg}^{-1} \mathrm{day}^{-1}\right)=\Sigma\left[\left(N_{i} \times D_{i} \times \operatorname{METS}_{\mathrm{i}}\right) / 365\right]$

where $N_{i}$ is the number of times the activity was performed, $D_{i}$ is the average duration in hours of the activity, and 
METS $_{i}$ is the estimated energy cost of the activity (kJ $\left.\mathrm{kg}^{-1} \mathrm{~h}^{-1}\right)$.

Covariates Information on several covariates was obtained by questionnaire at baseline (1988). Age was calculated from birth and observation dates and was coded as a continuous variable. Cigarette smoking status was coded as never, current smoker and former smoker, and consumption of alcohol was coded as abstainer, 1-3 drinks per day, 4-5 drinks per day and $\geq 6$ drinks per day. A parental history of diabetes was obtained and coded as $0=$ none or $1=$ either parent had diabetes.

Ascertainment of incident diabetes The presence of diabetes was assessed by a questionnaire during the 2002-2004 survey. Participants were asked to provide information on long-term health conditions that had lasted or were expected to last 6 months or more and that had been diagnosed by a health professional. Participants were coded as having diabetes if they answered 'yes' to the questions 'Do you suffer from diabetes?' or 'Do you currently take insulin?'. Since participants were aged at least 18 years at baseline and existing diabetes cases were screened out, incident cases were considered to be cases of type 2 diabetes. Given the low incidence rate of type 1 by comparison with type 2 diabetes among adults, any misclassification would have a minimal impact on the results.

Statistical analysis Logistic regression was used to predict incident diabetes from indicators of adiposity, physical activity and physical fitness at baseline. All models included age and sex as covariates, while additional models included the effects of smoking status, alcohol consumption, and parental history of diabetes. Given the strong correlations among the predictor variables, separate models were used to evaluate their predictive capacity. Receiver operating characteristic (ROC) curve analysis was used to evaluate the performance of the individual predictors of incident diabetes. An ROC curve is a plot of sensitivity (true positive fraction) versus 1 minus specificity (false positive fraction). The areas under the ROC curves were calculated along with $95 \%$ confidence intervals, which theoretically range from 0.5 (no predictive ability) to 1.0 (perfect prediction). Optimal thresholds for each variable were estimated as the value producing the best combination of sensitivity and specificity. Data management and logistic regression analyses were conducted using SAS software version 8.02 (SAS, Cary, NC, USA), and the ROC curves were generated using SPSS version 12.0 (SPSS, Chicago, IL, USA).

\section{Results}

The mean age at baseline was 36.8 years in men and 37.5 years in women (Table 1). Table 2 presents the correlations among the predictor variables at baseline. As expected, the indicators of adiposity were positively correlated with each other and negatively correlated with the indicators of physical fitness, with the exception of grip strength. Physical activity was positively correlated with maximal METs and indicators of MSF, but not with adiposity. The indicators of MSF were positively correlated with each other and the MSF composite score, as well as with maximal METs.

There were 78 new cases of diabetes (37 in men, 41 in women) in the cohort over the follow-up period, yielding a 15.5 year cumulative incidence of $5.0 \%(5.2 \%$ in men, $4.9 \%$ in women). All indicators of adiposity were positively associated with the incidence of diabetes (Table 3), with

Table 2 Pearson correlations among predictor variables at baseline (1988) in the Physical Activity Longitudinal Study

\begin{tabular}{|c|c|c|c|c|c|c|c|c|c|c|}
\hline & BMI & WC & WHR & SF5 & $\begin{array}{l}\text { Physical } \\
\text { activity }\end{array}$ & $\begin{array}{l}\text { Maximal } \\
\text { METs }\end{array}$ & $\begin{array}{l}\text { Sit- } \\
\text { ups }\end{array}$ & $\begin{array}{l}\text { Push- } \\
\text { ups }\end{array}$ & $\begin{array}{l}\text { Grip } \\
\text { strength }\end{array}$ & $\begin{array}{l}\text { Trunk } \\
\text { flexibility }\end{array}$ \\
\hline Waist circumference & $0.83^{\mathrm{a}}$ & & & & & & & & & \\
\hline WHR & $0.50^{\mathrm{a}}$ & $0.85^{\mathrm{a}}$ & & & & & & & & \\
\hline $\begin{array}{l}\text { Sum of skinfold } \\
\text { thicknesses (SF5) }\end{array}$ & $0.61^{\mathrm{a}}$ & $0.35^{\mathrm{a}}$ & 0.03 & & & & & & & \\
\hline Physical activity & $-0.07^{\mathrm{b}}$ & 0.004 & 0.04 & $-0.15^{\mathrm{a}}$ & & & & & & \\
\hline Maximal METs & $-0.34^{\mathrm{a}}$ & $-0.14^{\mathrm{a}}$ & $0.09^{\mathrm{b}}$ & $-0.55^{\mathrm{a}}$ & $0.17^{\mathrm{a}}$ & & & & & \\
\hline Sit-ups & $-0.26^{\mathrm{a}}$ & $-0.17^{\mathrm{a}}$ & -0.02 & $-0.43^{\mathrm{a}}$ & $0.24^{\mathrm{a}}$ & $0.64^{\mathrm{a}}$ & & & & \\
\hline Push-ups & $-0.18^{\mathrm{a}}$ & $-0.17^{\mathrm{a}}$ & -0.04 & $-0.40^{\mathrm{a}}$ & $0.21^{\mathrm{a}}$ & $0.52^{\mathrm{a}}$ & $0.63^{\mathrm{a}}$ & & & \\
\hline Grip strength & $0.25^{\mathrm{a}}$ & $0.52^{\mathrm{a}}$ & $0.59^{\mathrm{a}}$ & $-0.33^{\mathrm{a}}$ & $0.18^{\mathrm{a}}$ & $0.46^{\mathrm{a}}$ & $0.35^{\mathrm{a}}$ & $0.32^{\mathrm{a}}$ & & \\
\hline Trunk flexibility & $-0.18^{\mathrm{a}}$ & $-0.31^{\mathrm{a}}$ & $-0.29^{\mathrm{a}}$ & $-0.11^{\mathrm{a}}$ & $0.13^{\mathrm{a}}$ & $0.15^{\mathrm{a}}$ & $0.26^{\mathrm{a}}$ & $0.24^{\mathrm{a}}$ & $-0.09^{\mathrm{b}}$ & \\
\hline MSF score & $-0.24^{\mathrm{a}}$ & $-0.30^{\mathrm{a}}$ & $-0.23^{\mathrm{a}}$ & $-0.34^{\mathrm{a}}$ & $0.23^{\mathrm{a}}$ & $0.49^{\mathrm{a}}$ & $0.78^{\mathrm{a}}$ & $0.79^{\mathrm{a}}$ & $0.25^{\mathrm{a}}$ & $0.62^{\mathrm{a}}$ \\
\hline
\end{tabular}

${ }^{\mathrm{a}} p<0.001,{ }^{\mathrm{b}} p<0.05$ 
Table 3 Results of logistic regression analysis of adiposity, physical fitness and physical activity predicting the 15.5-year incidence of diabetes in the Physical Activity Longitudinal Study

\begin{tabular}{lllll}
\hline & $\mathrm{OR}^{\mathrm{a}}$ & $95 \% \mathrm{CI}$ & $\mathrm{OR}^{\mathrm{b}}$ & $95 \% \mathrm{CI}$ \\
\hline BMI & 2.02 & $1.63-2.50$ & 1.99 & $1.61-2.48$ \\
Waist circumference & 2.56 & $1.93-3.43$ & 2.53 & $1.89-3.42$ \\
WHR & 3.00 & $2.03-4.46$ & 2.94 & $1.98-4.38$ \\
Sum of skinfold thicknesses & 2.14 & $1.61-2.85$ & 2.09 & $1.56-2.79$ \\
Physical activity & 0.77 & $0.58-1.00$ & 0.78 & $0.59-1.02$ \\
Maximal METs & 0.28 & $0.14-0.57$ & 0.30 & $0.14-0.60$ \\
Sit-ups & 0.40 & $0.26-0.63$ & 0.41 & $0.26-0.64$ \\
Push-ups & 0.47 & $0.27-0.77$ & 0.49 & $0.28-0.81$ \\
Grip strength & 0.62 & $0.33-1.19$ & 0.62 & $0.33-1.20$ \\
Trunk flexibility & 0.71 & $0.48-1.03$ & 0.70 & $0.48-1.02$ \\
Musculoskeletal fitness & 0.38 & $0.23-0.62$ & 0.39 & $0.23-0.63$ \\
composite score & & & & \\
\hline
\end{tabular}

Results for each variable are from a separate model and are expressed per standard deviation of the variable

${ }^{a}$ Adjusted for age and sex

${ }^{\mathrm{b}}$ Adjusted for age, sex, smoking status, alcohol consumption and parental history of diabetes

odds ratios (ORs) ranging from 2.02 for $\mathrm{BMI}$ to 3.00 for WHR, from models adjusted for age and sex. Physical activity was associated with $23 \%$ lower odds of developing diabetes, and maximal METs was also associated with significantly lower odds of developing diabetes $(\mathrm{OR}=0.28)$. With the exception of grip strength and trunk flexibility, the indicators of MSF were associated with significantly lower odds of developing diabetes, with ORs ranging from 0.47 for push-ups to 0.38 for the MSF composite score. The inclusion of additional covariates had little effect on the results for any of the variables.

Table 4 and Fig. 1 present the results of the ROC analyses for the prediction of incident diabetes. All predictors, with the exception of physical activity and grip strength, had areas under the curve that were significantly different from 0.5 (the null hypothesis). Figure 1 presents the ROC curves for selected predictors. The similarity in the shape of the curves and the overlapping 95\% confidence intervals for the areas under the curve suggest that the significant indicators of adiposity and physical fitness have similar utility in the prediction of incident diabetes, and that no single indicator stands out as being superior to the others.

\section{Discussion}

The results of this study indicate that adiposity and physical fitness are both important predictors of incident diabetes, independently of several other risk factors, such as age, smoking, alcohol consumption and a parental history of diabetes. These results are in agreement with other studies that have documented relationships between adiposity or physical fitness and the development of diabetes [10-12]. A recent review has indicated that although both body weight and physical activity predict the development of diabetes, body weight (or BMI) plays a much greater role [6]. The results of the present study indicate that indeed adiposity was a better predictor of incident diabetes than physical activity; however, indicators of physical fitness predicted diabetes equally well. Thus, perhaps some of the discrepant results may be related to the use of measures of physical activity versus physical fitness.

There are several potential explanations for the different results obtained when using physical activity versus physical fitness. First, the measures of CRF and MSF used in this study may be better markers for habitual levels of physical activity than the level of physical activity selfreported by questionnaire, which is a rather subjective measure that is influenced by recall errors and bias. Indeed, CRF is a stronger predictor than physical activity for a variety of health outcomes [13]. Second, perhaps the measures of CRF and MSF have captured another component of health unrelated to physical activity per se. Both CRF and MSF are influenced by the effects of genes [14],

Table 4 Results of ROC curve analysis predicting incident diabetes in participants in the Physical Activity Longitudinal Study

\begin{tabular}{|c|c|c|c|c|c|}
\hline \multirow{2}{*}{$\begin{array}{l}\text { Variable } \\
\text { BMI }\left(\mathrm{kg} / \mathrm{m}^{2}\right)\end{array}$} & \multicolumn{2}{|c|}{ Area under the curve $(95 \% \mathrm{CI})$} & \multirow{2}{*}{$\frac{\text { Optimal threshold }}{25.6}$} & \multirow{2}{*}{$\frac{\text { Sensitivity }(\%)}{66.1}$} & \multirow{2}{*}{$\frac{\text { Specificity }(\%)}{66.1}$} \\
\hline & 0.71 & $(0.64,0.79)$ & & & \\
\hline Waist circumference $(\mathrm{cm})$ & 0.72 & $(0.64,0.80)$ & 85.5 & 66.7 & 66.7 \\
\hline WHR & 0.70 & $(0.62,0.77)$ & 0.85 & 63.2 & 63.2 \\
\hline Sum of skinfolds (mm) & 0.66 & $(0.59,0.74)$ & 74.2 & 60.4 & 61.3 \\
\hline Physical activity $\left(\mathrm{kJ} \mathrm{k}^{-1}\right.$ day $\left.^{-1}\right)$ & 0.56 & $(0.50,0.63)$ & 6.07 & 55.7 & 58.8 \\
\hline Maximal METs & 0.63 & $(0.55,0.71)$ & 11.3 & 60.6 & 59.0 \\
\hline Sit-ups $(n / \mathrm{min})$ & 0.70 & $(0.61,0.79)$ & 20.5 & 66.7 & 66.0 \\
\hline Push-ups (n) & 0.65 & $(0.55,0.74)$ & 13.5 & 56.3 & 58.6 \\
\hline Grip strength $(\mathrm{kg})$ & 0.52 & $(0.43,0.62)$ & 73.9 & 52.9 & 50.8 \\
\hline Trunk flexibility (cm) & 0.63 & $(0.54,0.73)$ & 26.4 & 61.8 & 62.0 \\
\hline Musculoskeletal fitness composite score & 0.74 & $(0.64,0.84)$ & -0.58 & 73.3 & 73.3 \\
\hline
\end{tabular}




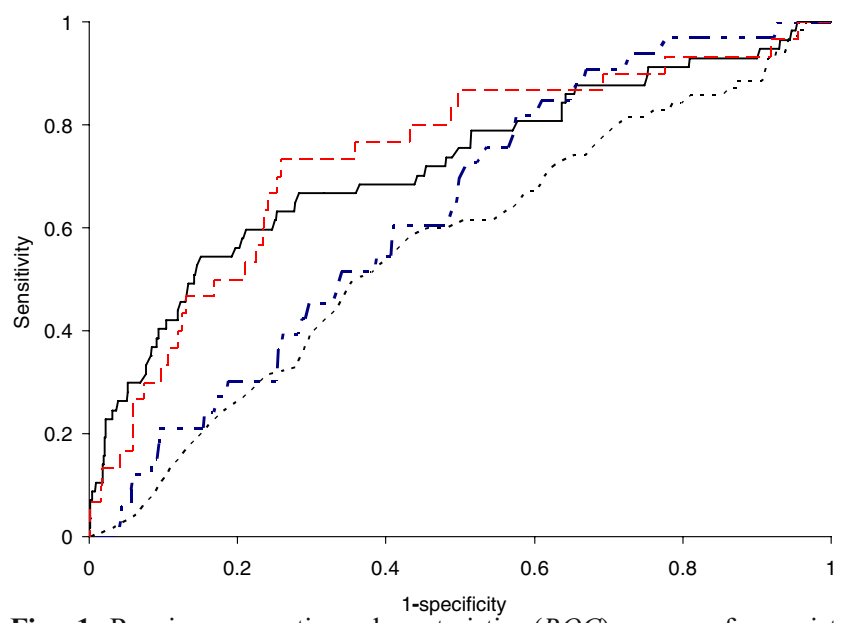

Fig. 1 Receiver operating characteristic (ROC) curves for waist circumference (solid black line), physical activity (short dashed black line), maximal METs (intermittently dotted blue line), and musculoskeletal fitness composite score (red dashed line) for the prediction of incident diabetes in the Physical Activity Longitudinal Study

and it is possible that the same underlying genes may be responsible for providing some innate health qualities. Third, the sample size in the present study may not have been sufficient to allow a significant effect of physical activity to be detected. The variance associated with the measure of physical activity is much larger than that for the measures of CRF and MSF. Thus, although the OR for physical activity indicated a $22 \%$ lower risk of incident diabetes, it was not statistically significant. Nevertheless, the fact that the indicators of physical fitness were predictive of incident diabetes suggests that the promotion of physical activity is important, as the main strategy used to improve physical fitness levels is to increase physical activity.

To our knowledge, this is the first study to examine the relationship between MSF and incident diabetes. The results indicate that measures of muscular strength (grip strength) and endurance (sit-ups and push-ups) were significantly related to the incidence of diabetes. Although their results are not directly comparable with ours, Jurca et al. [15] have recently reported a significant association between muscular strength and the incidence of the metabolic syndrome in men, defined according to National Cholesterol Education Program criteria. Over an average of 6.7 years of follow-up, the relative risk of developing metabolic syndrome across successive quartiles of muscular fitness decreased significantly (hazard ratio $=1.0,0.93,0.89$, 0.66 ; $p$ for trend $=0.004)$. Taken together with the results of the present study, these results highlight the importance of MSF in the prediction of metabolic disorders. Further research is required to better delineate the relationships between resistance exercise, MSF and the development of diabetes.
Few previous studies have documented relationships between CRF and incident diabetes [16-19]. The earliest study on this topic, conducted among Finnish men, reported that the multivariate-adjusted odds of developing type 2 diabetes over 4 years decreased across successive quartiles of $\mathrm{CRF}$ (OR=1.0 [reference], 0.77, 0.26, 0.15) [15]. Similarly, a recent study among Japanese men reported that the multivariate-adjusted relative risk of developing type 2 diabetes over 14 years decreased across successively higher quartiles of $\mathrm{CRF}$ (relative risk $=1.0$ [reference], 0.78, $0.63,0.56 ; p$ for trend=0.001) [19]. Further, over an average of 6 years of follow-up in the Aerobics Center Longitudinal Study, the multivariate-adjusted risk of type 2 diabetes in men was 3.7 times higher in the low-fitness group by comparison with the high-fitness group [17]. Finally, in the only study to include women as participants, the risk of developing diabetes over 15 years of follow-up was three to six times higher in the low-fitness by comparison with the high-fitness young adults in the Coronary Artery Risk Development in Young Adults (CARDIA) study [18]. In summary, all of the published studies of CRF and diabetes, including the present study, have demonstrated remarkably consistent results, showing that higher levels of CRF result in a lower incidence of diabetes.

This study has several strengths and limitations that warrant discussion. A marked strength is the availability of a large battery of directly measured indicators of adiposity and fitness. Another strength of the study is the longitudinal cohort, which is based on a representative sample of the Canadian population at baseline. A limitation is the reliance on self-reported physician-diagnosed diabetes at follow-up, as we were unable to obtain measurements of fasting glucose or to confirm the self-reported data. An analysis of data from the Manitoba Heart Health Survey indicated that undiagnosed diabetes cases accounted for about one-third of all diabetes cases [20]. Further, the analyses reported in this paper are based on 1,543 individuals with the necessary data at baseline and at follow-up. However, this represents only about a third of the entire PALS cohort $(n \sim 4,900)$. There was a $29.8 \%$ non-response rate in the $2002-2004$ survey $(10 \%$ were lost to follow-up and $19.8 \%$ refused to participate), and it is projected that $18.3 \%(n=899)$ of the participants are deceased [7]. The causes of death among the decedents are unknown at this time; however, it is likely that some deaths were due to complications associated with diabetes. However, data from the longitudinal National Population Health Survey (1994-1998) documented a 4year cumulative incidence rate of 1.4 new cases of diabetes per 100 people in subjects aged $\geq 20$ years [21]. Extrapolating these results to 15.5 years of follow-up yields an expected incidence of 5.4 cases per 100 people, or 83 new cases per 1,543 people. This compares very well to the observed 78 cases of incident diabetes in our study. Thus, 
the incidence of diabetes in this cohort is similar to that observed in the Canadian adult population.

In summary, this study has documented significant relationships between both adiposity and physical fitness and the development of diabetes in a Canadian population. Although the predictive relationships differed somewhat across different variables, the underlying behaviour that is associated with reductions in adiposity and increases in physical fitness is physical activity [22]. The level of physical activity associated with lower health risks in this and other studies can be achieved by following the current public health recommendations, which are to accumulate at least 30 min of moderate-intensity physical activity on most days of the week [23]. The results suggest that interventions that increase physical activity levels, and in particular those that target weight loss, would be most effective in the prevention of diabetes. Indeed, results from the Diabetes Prevention Program and Diabetes Prevention Study have demonstrated significantly lower incidence rates for diabetes among overweight individuals with impaired glucose tolerance participating in interventions that included physical activity counselling and a targeted weight loss compared with controls $[24,25]$. These results need to be replicated in samples of the healthy population in order to better characterise the long-term prevention of diabetes in the population.

Acknowledgements This work was funded by a grant from the Social Sciences and Humanities Research Council of Canada and the Canadian Institutes of Health Research (Strategic Joint Initiative Grant on Society, Culture and the Health of Canadians II Grant No 8392000-1032). The 1981 Canada Fitness Survey was supported by Fitness and Amateur Sport (now the Physical Activity Unit of Health Canada) and the 1988 Campbell's Survey on Well-being in Canada was funded by Fitness and Amateur Sport, the National Health Research and Development Program of Health Canada (Grant No 6606-3217-46) and the Campbell Soup Company Ltd.

Duality of interest The authors have no conflicts of interest to disclose.

\section{References}

1. World Health Organization (2005) Preventing chronic disease: a vital investment. WHO, Geneva

2. Statistics Canada (2004) Health indicators, vol 2004, no 1. Catalogue No. 82-221. Statistics Canada, Ottawa, ON

3. Dawson KG, Gomes D, Gerstein H, Blanchard JF, Kahler KH (2002) The economic cost of diabetes in Canada, 1998. Diabetes Care 25:1303-1307

4. Health Canada (2003) Responding to the challenge of diabetes in Canada: first report of the National Diabetes Surveillance System (NDSS) 2003 Catalogue no. H39-4/21-2003E. Minister of Health, Ottawa, ON

5. Eyre H, Kahn R, Robertson RM et al (2004) Preventing cancer, cardiovascular disease, and diabetes: a common agenda for the
American Cancer Society, the American Diabetes Association, and the American Heart Association. Stroke 35:1999-2010

6. Weinstein AR, Sesso HD (2006) Joint effects of physical activity and body weight on diabetes and cardiovascular disease. Exerc Sport Sci Rev 34:10-15

7. Craig CL, Gauvin L, Cragg S et al (2005) Towards a social epidemiological perspective on physical activity and health: The aims, design and methods of the physical activity longitudinal study (PALS). J Phys Act Health 3:272-284

8. Fitness Canada (1981) Standardized test of fitness: operations manual, 2nd edn. Ministry of Fitness and Amateur Sport, Ottawa, ON

9. Taylor HL, Jacobs DR, Schucker B, Knudsen J, Leon AS, Debacker G (1978) A questionnaire for leisure time physical activities. J Chronic Dis 31:741-755

10. Astrup A, Finer N (2000) Redefining type 2 diabetes: 'diabesity' or 'obesity dependent diabetes mellitus'. Obes Rev 1:57-59

11. Bassuk SS, Manson JE (2005) Epidemiological evidence for the role of physical activity in reducing risk of type 2 diabetes and cardiovascular disease. J Appl Physiol 99:1193-1204

12. LaMonte MJ, Blair SN, Church TS (2005) Physical activity and diabetes prevention. J Appl Physiol 99:1205-1213

13. Blair SN, Jackson AS (2001) Physical fitness and activity as separate heart disease risk factors: a meta-analysis. Med Sci Sports Exerc 33:762-764

14. Wolfarth B, Bray MS, Hagberg JM et al (2005) The human gene map for performance and health-related fitness phenotypes: the 2004 update. Med Sci Sports Exerc 37:881-903

15. Jurca R, LaMonte MJ, Barlow CE, Kampert JB, Church TS, Blair SN (2005) Association of muscular strength with incidence of metabolic syndrome in men. Med Sci Sports Exerc 37:1849 1855

16. Lynch J, Helmrich SP, Lakka TA et al (1996) Moderately intense physical activities and high levels of cardiorespiratory fitness reduce the risk of non-insulin-dependent diabetes mellitus in middle-aged men. Arch Intern Med 156:1307-1314

17. Wei M, Gibbons LW, Mitchell TL, Kampert JB, Lee CD, Blair SN (1999) The association between cardiorespiratory fitness and impaired fasting glucose and type 2 diabetes mellitus in men. Ann Intern Med 130:89-96

18. Carnethon MR, Gidding SS, Nehgme R, Sidney S, Jacobs DR, Liu K (2003) Cardiorespiratory fitness in young adulthood and the development of cardiovascular disease risk factors. JAMA 290:3092-3100

19. Sawada SS, Lee IM, Muto T, Matuszaki K, Blair SN (2003) Cardiorespiratory fitness and the incidence of type 2 diabetes: prospective study of Japanese men. Diabetes Care 26:29182922

20. Young TK, Mustard CA (2001) Undiagnosed diabetes: does it matter? Can Med Assoc J 164:24-28

21. Health Canada (2002) Diabetes in Canada, 2nd edn. Center for Chronic Disease Prevention and Control, Population and Public Health Branch, Health Canada, Ottawa, ON

22. Blair SN, Church TS (2004) The fitness, obesity, and health equation: is physical activity the common denominator? JAMA 292:1232-1234

23. US Department of Health and Human Services (1996) Physical activity and health: a report of the Surgeon General. Department of Health and Human Services, Centers for Disease Control and Prevention, National Center for Chronic Disease Prevention and Health Promotion, Atlanta, GA

24. Eriksson J, Lindstrom J, Valle TT et al (1999) Prevention of type 2 diabetes in subjects with impaired glucose tolerance: the Diabetes Prevention Study (DPS) in Finland. Diabetologia 42:793-801

25. Knowler WC, Barrett-Connor E, Fowler SE et al (2002) Reduction in the incidence of type 2 diabetes with lifestyle intervention or metformin. N Engl J Med 346:393-403 